\title{
Microwave resonant absorption of viruses through dipolar coupling with confined acoustic vibrations
}

\author{
Tzu-Ming Liu ${ }^{1}$ Hung-Ping Chen, ${ }^{1}$ Li-Tzu Wang, ${ }^{2}$ Jen-Ren Wang, ${ }^{2}$ Tang-Nian Luo, ${ }^{3}$ \\ Yi-Jan Chen, ${ }^{3}$ Shen-Iuan Liu, ${ }^{3}$ and Chi-Kuang Sun ${ }^{1,3,4, a)}$ \\ ${ }^{1}$ Graduate Institute of Photonics and Optoelectronics, National Taiwan University, Taipei 10617, Taiwan \\ ${ }^{2}$ Department of Medical Laboratory Science and Biotechnology, National Cheng Kung University, \\ Tainan 70101, Taiwan \\ ${ }^{3}$ Department of Electrical Engineering, National Taiwan University, Taipei 10617, Taiwan \\ ${ }^{4}$ Research Center for Applied Sciences, Academia Sinica, Taipei 10617, Taiwan
}

(Received 3 November 2008; accepted 4 January 2009; published online 27 January 2009)

\begin{abstract}
In this letter, with an electric double layer on the surface of spherical viruses, we confirm that one of the microwave resonant absorption (MRA) mechanisms of viruses is through dipolar coupling with confined acoustic vibrations. By treating spherical virions as free homogeneous nanoparticles, we found that the MRA frequencies agree well with that of $l=1$ dipolar modes predicted by the elastic continuum theory. The magnitude of MRA was also found to change with the amount of adsorbed charges on the surface of virions. Our results provide a method to observe three-dimensionally confined acoustic vibrations in biological systems. (C) 2009 American Institute of Physics. [DOI: 10.1063/1.3074371]
\end{abstract}

When confined acoustic vibrations ${ }^{1}$ modify dipole moments of nanoparticles, incoming electromagnetic waves oscillating at the same frequencies will resonantly couple with the confined acoustic modes and result in terahertz photon absorption. ${ }^{2,3}$ For nonpiezoelectric materials, the occurrence of such resonant absorption requires core-shell charge separation in nanoparticles. ${ }^{2,4}$ Intriguingly, in biological systems, most proteins carry charges in buffer solutions. Counterions dissolved in solution will thus be adsorbed on the surface of a protein and form an electric double layer. This charge separation structures pave the roads for the occurrence of electric oscillations $^{5,6}$ and can be exploited to investigate the confined acoustic vibrations in biological systems. Decades ago, Edwards et al. ${ }^{7}$ reported resonant microwave absorption corresponding to longitudinal standing waves along linear DNA molecules. It was proposed that a hydration layer surrounding DNA molecules could lower the viscoelastic transition frequency, raise the quality factor of confined acoustic vibrations, and result in a microwave resonant absorption (MRA). ${ }^{8}$ Except for one-dimensional standing acoustic waves, in biological systems, two-dimensional-radial confined acoustic vibrations on M13 phages ${ }^{9}$ and virus-inorganic nanotubes ${ }^{10}$ were also investigated through low-frequency Raman scattering. By far, there is no report on the observation of three-dimensionally (3D) confined acoustic vibrations in biological systems. In this work, through microwave absorption measurements, we report the observation of 3D confined acoustic vibrations in spherical virions. With a rough estimation by the theory of Lamb, ${ }^{1}$ the measured MRA frequencies in the studied viruses were found to agree well with that of the spheroidal (SPH) $(l=1)$ modes. By changing the $p \mathrm{H}$ values of solvents, the observed MRAs of viruses were found to be enhanced with more adsorbing counterions and suppressed by the aggregation of virions, confirming the pro-

\footnotetext{
a) Author to whom correspondence should be addressed. Electronic mail: sun@cc.ee.ntu.edu.tw.
}

posed mechanism. Our results imply a scenario for microwaves interaction with biological systems.

According to the derivation of Lamb ${ }^{1}$ and the group symmetry argument, ${ }^{11}$ among all 3D-quantized acoustic modes, the dipolar active one is the SPH mode with an angular momentum $l=1$. Due to its Raman inactive nature, the $l=1 \mathrm{SPH}$ mode has been rarely studied experimentally., Both the theoretically calculated [SPH, $l=1, n=0]$ and $[\mathrm{SPH}$, $l=1, n=1]$ dipolar modes have relative displacement between the core and the shell. ${ }^{4}$ Therefore, a core-shell charge structure is required for the activation of dipolar coupling in nanoparticles. ${ }^{2}$ The frequencies of the corresponding dipolar modes can be calculated by the eigenvalue equation ${ }^{1}$

$$
4 \frac{j_{2}(\xi)}{j_{1}(\xi)} \xi-\eta^{2}+2 \frac{j_{2}(\eta)}{j_{1}(\eta)} \eta=0
$$

where $\xi=2 \pi \nu R / V_{L}, \eta=2 \pi \nu R / V_{T}$, and $j_{l}(\eta)$ is the spherical Bessel function of the first kind; $R$ is the radius of nanoparticles and $V_{L}$ and $V_{T}$ are the sound velocities of longitudinal and transverse waves, respectively. Since most typical condensed matters have a $\sim 0.3$ Poisson's ratio, ${ }^{12}$ the $V_{L} / V_{T}$ ratio is kept around 2. The dipolar vibration frequencies of viruses are thus expected to be proportional to $V_{L} / R$ (or $V_{T} / R$ ). Even though the acoustic parameter of a total virus is rarely studied, most viruses are composed of lipids, proteins, and genomes. The $V_{L}$ of lipid is $1520 \mathrm{~m} / \mathrm{s} .{ }^{13}$ Most protein crystals' $V_{L}$ are around $1800 \mathrm{~m} / \mathrm{s} .{ }^{14}$ The reported $V_{L}$ of the uncompressed wet genomes is $\sim 1700 \mathrm{~m} / \mathrm{s} .{ }^{15}$ Due to the fact that most viruses have highly compressed genomes and their capsid proteins have strong tension, the effective $V_{L}$ of a total virus should thus be on the order of or higher than 1500 $1800 \mathrm{~m} / \mathrm{s}$ if the virus does not have a soft envelope. Recently, using a Brillouin scattering measurement, the effective $V_{L}$ of the $17 \mathrm{~nm}$ satellite tobacco mosaic virus (STMV) was determined to be $1920 \pm 70 \mathrm{~m} / \mathrm{s} .{ }^{16}$ Using these information, one can estimate the frequency of the [SPH, $l=1, n=0]$ mode for a $30 \mathrm{~nm}$ spherical virus to be around $30-40 \mathrm{GHz}$. 

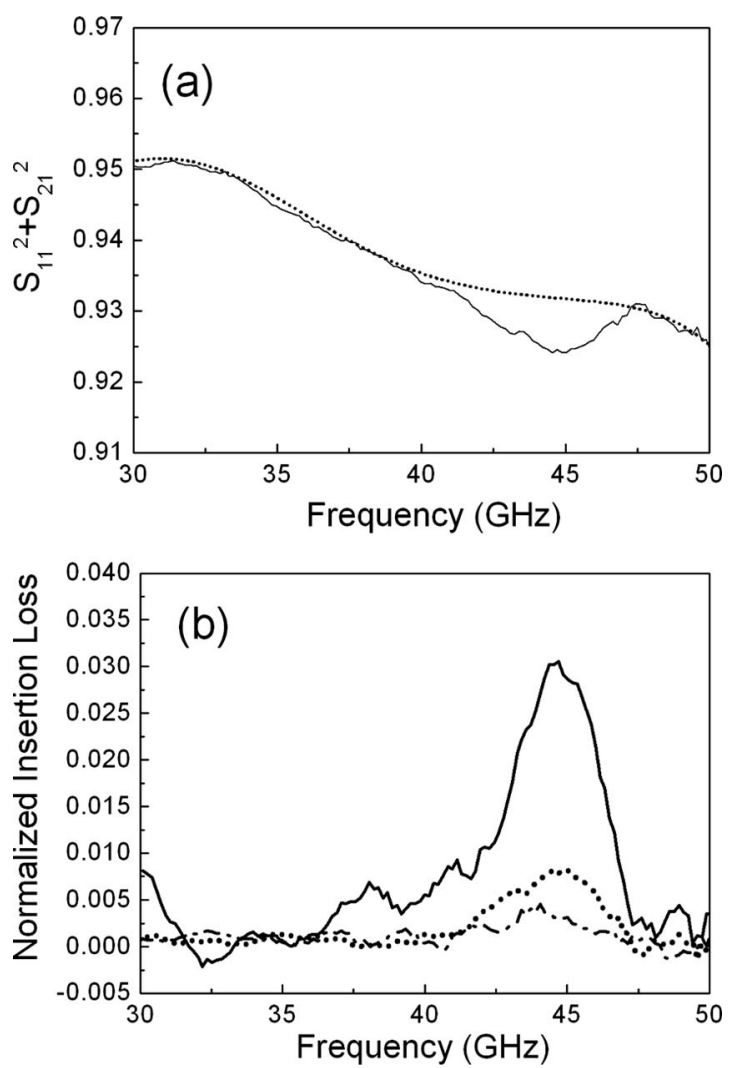

FIG. 1. (a) The microwave absorption spectra (solid curve) of enterovirus 71 after removing the buffer's contribution. The dotted curve is the background absorption fitted to the lower and higher frequency part of the original absorption spectrum. (b) The MRA spectrum of enterovirus 71 in $p \mathrm{H}$ $=5.4$ (dash-dotted curve), $p \mathrm{H}=6.4$ (solid curve), and $p \mathrm{H}=7.4$ (dotted curve) buffer. The contributions from buffer and background are removed.

To observe 3D-confined acoustic vibrations through MRA, we prepared isometric enterovirus 71 (EV71) (Ref. 17) and spherical influenza A viruses. To perform MRA spectral measurements, these viruses were cultured, isolated, purified, and then preserved in phosphate buffer saline (PBS) buffer liquids. In each measurement, we took $1 \mu \mathrm{l}$ viral solution by a micropipette and uniformly dropped it on the midst of a coplanar waveguide (CPW) circuit. The guided microwaves can thus incident on the virus-containing solution. The reflection $S_{11}$ and transmission $S_{21}$ parameters of the CPW circuit were simultaneously recorded with a standard $40 \mathrm{MHz}-65 \mathrm{GHz}$ network analyzer. The microwave attenuation spectra can be evaluated by $\left|S_{11}\right|^{2}+\left|S_{21}\right|^{2}$. As a control, we also measured the attenuation spectrum spectra of the corresponding buffer liquids with the same volume on the same device. By comparing the attenuation spectra of the buffer solutions with and without viruses, the corresponding microwave attenuation spectra of viruses $A_{v}(f)$ can be obtained.

Under test EV71 virions have a diameter of 28.5 \pm $1.5 \mathrm{~nm}$ (Ref. 18) and were purified and inactivated with $1 \mathrm{ml}$ PBS $(p \mathrm{H}=7.4)$ containing $1 \%$ glutaraldehyde. The corresponding zeta potential of EV71 virions was $-25 \mathrm{mV}$, and the hydrodynamic diameter $(40 \pm 2.2 \mathrm{~nm})$ was slightly larger than that characterized by transmission electron microscopy (TEM). ${ }^{18}$ The discrepancy in diameter could be due to the adsorbed hydration layers or the electric double layers. ${ }^{19}$ However, most of these adsorbed counterions or water molecules have weak and loose adsorption; only a thin

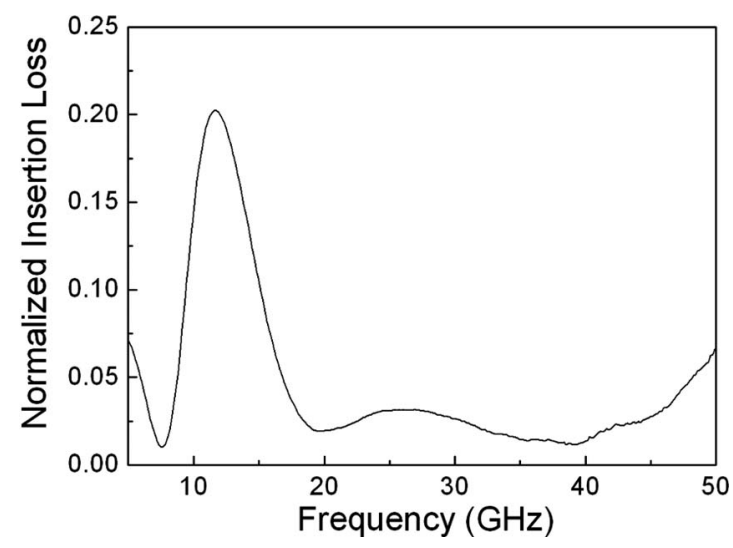

FIG. 2. The MRA spectrum of influenza A viruses after removing the buffer's and the background's contribution.

(1-2 nm) layer has strong and fixed attachment on the surface of virions. ${ }^{20}$ Therefore, the actual sizes to be considered in collective motions and theoretical calculations should be very close to those under TEM. After making sure the dispersion of EV71 virions in PBS buffer, we measured the microwave attenuation spectrum of EV71 [Fig. 1(a), solid curve] and found an absorption dip around $45 \mathrm{GHz}$. With a frequency close to the above mentioned estimation (30-40 $\mathrm{GHz}$ ), this observed resonant absorption could thus be contributed from the MRA through the [SPH, $l=1, n=0]$ mode of EV71 with an effective $V_{L}$ of $2450 \mathrm{~m} / \mathrm{s}$. Because the genomic base number of EV71 (7.5 kb) (Ref. 21) is approximately six times that of STMV (1.24 kb) (Ref. 22) while the volume only expands $\sim 4.7$ times, the increase in sound velocity could be due to the stiffening of virions by more genomes packaged inside the capsids. To extract the actual amount of absorption related to confined acoustic modes, we normalized $A_{v}(f)$ to the nonresonant background absorption $A_{\mathrm{vb}}(f)$ [Fig. 1(a), dotted curve] and calculated the normalized insertion loss $\left[1-A(f) / A_{b}(f)\right]$ of EV71 [Fig. 1(b), dotted curve]. The peak insertion loss contributed by confined acoustic vibrations was $0.8 \%$. Because the transverse velocity of water is zero and the displacement vectors of the $[\mathrm{SPH}$, $l=1, n=0]$ mode have shear components on the surface, ${ }^{4}$ the corresponding acoustic impedance contrast between water and EV71 virions should be higher than that of the $[\mathrm{SPH}$, $l=0]$ modes. ${ }^{14}$ Moreover, there are hydration layers on the surface of EV71, which make the solvent reactive rather than resistive. ${ }^{8}$ As we expected, the measured $45 \mathrm{GHz}$ MRA peak has a MRA quality factor $Q_{\mathrm{MRA}}=10$ in the water surrounding. The exact cause of the resonant absorption bandwidth broadening is not fully understood. It could originate from homogeneous broadening of acoustic quality factor or inhomogeneous size variation of virions.

To further confirm if the observed absorption peak is through the dipolar coupling with confined acoustic modes, not from the rotation of solvent molecules, ${ }^{23}$ we measured the attenuation spectra of spherical influenza A viruses. The diameters of influenza A virions are typically $100 \mathrm{~nm}$ (Ref. 24) and can vary from 80 to $120 \mathrm{~nm}^{25}$ The influenza A virions were purified and inactivated with $1 \mathrm{ml}$ PBS $(p \mathrm{H}$ $=7.4$ ) containing $1 \%$ glutaraldehyde. The virions had -11 $\mathrm{mV}$ zeta potential and $93 \pm 5 \mathrm{~nm}$ hydrodynamic diameter, indicating no aggregation effect. As shown in Fig. 2, we observed a resonant absorption frequency peak around $12 \mathrm{GHz}$, reflecting the expected size dependency and further 
confirming our finding that confined dipolar acoustic vibrations of virions can indeed cause MRA. Considering that influenza A virus's effective $V_{L}$ is close to that of EV71, this MRA frequency together with that of EV71 follows the theoretically predicted $1 / R$ dependency. Another very interesting finding is that the MRA related to the high-order [SPH, $l=1, n=1]$ acoustic mode, which is supposed to be twice the frequency of the [SPH, $l=1, n=0]$ acoustic mode under the homogeneous sphere assumption, also appears around 26 $\mathrm{GHz}$ (see Fig. 2). It does not only further support the fact that we are indeed observing the confined acoustic modes, but it also validates the dipolar activity of the high-order [SPH, $l=1]$ mode. The slight deviation from the ideal value could be due to the inhomogeneous and complex structures of influenza A virus. ${ }^{25}$ We are not able to confirm if this high-order mode also exists in the MRA spectra of the EV71 due to limited frequency range (up to $65 \mathrm{GHz}$ ) of the adopted network analyzer.

For a final confirmation if the proposed dipolar interaction is responsible for the MRA, we alter the charge status on the surface of EV71 virions and investigate the corresponding microwave attenuation spectra. Purified EV71 virions were resuspended in $p \mathrm{H}=5.4$ and 6.4 PBS buffer separately. Their concentrations of virions were the same as that in our previous investigation. The corresponding zeta potentials of the virions were -3 and $-11.8 \mathrm{mV}$, respectively. This trend, together with the previous $p \mathrm{H}=7.4$ case, reveals that $p \mathrm{H}$ $=5.4$ is close to EV71's isoelectric point, and an aggregation might happen under such a condition. As we suspected, we found the EV71 virions had 720nm hydrodynamic diameter in the $p \mathrm{H}=5.4$ solution, which indicated aggregation in solution. The other one $(p \mathrm{H}=6.4)$ showed diameters of $35 \pm 2 \mathrm{~nm}$, indicating full dispersion with a thick hydration layer or electric double layer on the surface of the virions. ${ }^{19}$ The virions in the $p \mathrm{H}=6.4$ solution had smaller absolute value of zeta potential than the $p \mathrm{H}=7.4$ case by adsorbing more positive ions on the surface. The adsorption of more charges on the surface will thus create a better environment for the required core-shell charge separation structure and should increase the resonant dipolar coupling with the confined acoustic modes. ${ }^{2,3}$ Then we measured their microwave attenuation spectra and calculated their normalized insertion loss [Fig. 1(b)]. Compared with the $p \mathrm{H}=7.4$ case, the peak $45 \mathrm{GHz}$ MRA loss of EV71 in the $p \mathrm{H}=6.4$ solution was found to be enhanced from $0.8 \%$ to $3 \%$, agreeing once again with the proposed dipolar coupling mechanism. In the $p \mathrm{H}$ $=5.4$ solution, although more positive ions adsorbed on the surface of EV71, aggregation of virions will, on the contrary, suppress the magnitude of insertion loss. This could be the reason why the MRA signal for the $p \mathrm{H}=5.4$ case was reduced to the noise level. These results of EV71 imply that the charge status and the dispersion condition are crucial for the observation of confined acoustic modes in biological systems, which has never been discussed and investigated in previous debates of MRA in selected DNA., , $826-28^{2}$

In summary, through the dipolar coupling to confined acoustic modes, we observed the MRA of virions. The MRA frequencies agree well with that of $l=1$ dipolar modes predicted by the elastic continuum theory. When the size of virions changed, they also followed the theoretically predicted $1 / R$ dependency. By changing the $p \mathrm{H}$ value of solutions, we manipulated the amount of adsorbed charges, and the MRA will be either enhanced with more adsorbed charges or suppressed when virions reach isoelectric points. Our results not only observed 3D confined acoustic vibrations in virions but also imply that charge status and dispersion condition are critical for the presence of MRA in biological systems.

This project has been sponsored by the National Science Council of Taiwan under Grant Nos. NSC97-2120-M-002010, NSC 97-2221-E-002-047-MY3, and NSC 96-2628-E002-043-MY3 and by the National Taiwan University Research Center for Medical Excellence. We thank D. B. Murray and L. Saviot for technical discussions on the dipolar modes in a nanosphere.

${ }^{1}$ H. Lamb, Proc. London Math. Soc. 13, 189 (1881).

${ }^{2}$ T.-M. Liu, J.-Y. Lu, H.-P. Chen, C.-C. Kuo, M.-J. Yang, C.-W. Lai, P.-T. Chou, M.-H. Chang, H.-L. Liu, Y.-T. Li, C.-L. Pan, S.-H. Lin, C.-H. Kuan, and C.-K. Sun, Appl. Phys. Lett. 92, 093122 (2008).

${ }^{3}$ T.-M. Liu, M.-J. Yang, C.-W. Lai, P.-T. Chou, M.-H. Chang, H.-L. Liu, and C.-K. Sun, Phys. Rev. B 77, 085428 (2008).

${ }^{4}$ D. B. Murray, C. H. Netting, L. Saviot, C. Pighini, N. Millot, D. Aymes, and H.-L. Liu, J. Nanoelectron. Optoelectron. 1, 92 (2006).

${ }^{5}$ H. Fröhlich, Int. J. Quantum Chem. 2, 641 (1968).

${ }^{6}$ W. Grundler and F. Keilmann, Phys. Rev. Lett. 51, 1214 (1983).

${ }^{7}$ G. S. Edwards, C. C. Davis, J. D. Saffer, and M. L. Swicord, Phys. Rev. Lett. 53, 1284 (1984)

${ }^{8}$ L. L. Van Zandt, Phys. Rev. Lett. 57, 2085 (1986).

${ }^{9}$ K. T. Tsen, E. C. Dykeman, O. F. Sankey, S.-W. D. Tsen, N.-T. Lin, and J. G. Kiang, J. Biomed. Opt. 12, 024009 (2007).

${ }^{10}$ V. A. Fonoberov and A. A. Balandin, Nano Lett. 5, 1920 (2005)

${ }^{11}$ E. Duval, Phys. Rev. B 46, 5795 (1992).

${ }^{12}$ M. H. Sadd, Elasticity: Theory, Applications and Numerics (Elsevier, Dordrecht, 2005).

${ }^{13}$ Y. Saijo, N. Hozumi, C. Lee, M. Nagao, K. Kobayashi, N. Oakada, N. Tanaka, E. S. Filho, H. Sasaki, M. Tanaka, and T. Yambe, Ultrasonics 44, e51 (2006).

${ }^{14}$ M. Talati and K. Jha, Phys. Rev. E 73, 011901 (2006).

${ }^{15}$ M. B. Hakim, S. M. Lindsay, and J. Powell, Biopolymers 23, 1185 (1984)

${ }^{16}$ B. Stephanidis, S. Adichtchev, P. Gouet, A. McPherson, and A. Mermet, Biophys. J. 93, 1354 (2007).

${ }^{17}$ J.-R. Wang, H.-P. Tsai, P.-F. Chen, Y.-J. Lai, J.-J. Yan, D. Kiang, K.-H. Lin, C.-C. Liu, and I.-J. Su, J. Clin. Virol. 17, 91 (2000).

${ }^{18}$ Y.-Y. Wen, T.-Y. Chang, S.-T. Chen, C. Li, and H.-S. Liu, J. Med. Virol 70, 109 (2003).

${ }^{19}$ R. Xu, Langmuir 14, 2593 (1998).

${ }^{20}$ J. Israelachvili and H. Wennerström, Nature (London) 379, 219 (1996).

${ }^{21}$ I. Merkle, M. J. M. van Ooij, F. J. M. van Kuppeveld, D. H. R. F. Glaudemans, J. M. D. Galama, A. Henke, R. Zell, and W. J. G. Melchers, J. Virol. 76, 9900 (2002).

${ }^{22}$ A. A. Brunt, K. Crabtree, M. J. Dallwitz, A. J. Gibbs, L. Watson, and E. J. Zurcher, Plant Viruses Online, 1996, http://image.fs.uidaho.edu/vide/ descr806.htm.

${ }^{23}$ S. J. Webb and A. D. Booth, Nature (London) 222, 1199 (1969).

${ }^{24}$ D. M. Knipe, P. M. Howley, D. E. Griffin, and R. A. Lamb, Field's Virology, 5th ed. (Lippincott Williams \& Wilkins, Philadelphia, 2006), Chap. 47.

${ }^{25}$ T. Noda, H. Sagara, A. Yen, A. Takada, H. Kida, R. H. Cheng, and Y. Kawaoka, Nature (London) 439, 490 (2006).

${ }^{26}$ C. Gabriel, E. H. Grant, R. Tata, P. R. Brown, B. Gestblom, and E. Noreland, Nature (London) 328, 145 (1987).

${ }^{27}$ K. R. Foster, B. R. Epstein, and M. A. Gealt, Biophys. J. 52, 421 (1987).

${ }^{28}$ I. J. Bigio, T. R. Gosnell, P. Mukherjee, and J. D. Saffer, Biopolymers 33, 147 (1993). 